\title{
1 Accounting for the role of turbulent flow on particle dislodgement via a 2 coupled quadrant analysis of velocity and pressure sequences
}

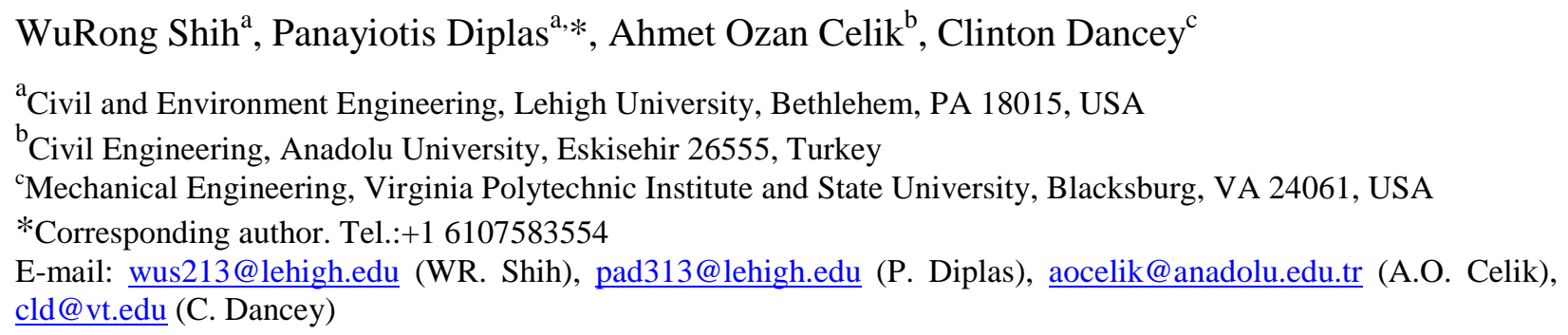

Abstract Recent studies have demonstrated the importance of duration, in addition to magnitude, of energetic turbulent events on particle dislodgement under threshold of motion conditions. It is therefore proposed here that the use of turbulence sequences, instead of single instantaneous extreme events, is more appropriate for characterizing the role of fluctuating velocities and associated hydrodynamic forces on particle mobility. The validity of the proposed method is examined through the detailed analysis of directly measured velocity and pressure data sets. In an effort to relate the results of the conventional velocity quadrant analysis with the instantaneous pressure measurements, the concept of pressure quadrant analysis is introduced. The coupled sequences of the synchronous velocity and pressure quadrant events allow for a more complete description of the local turbulent flow characteristics, including the prevalent coherent structures, and more clear interpretation of their role on particle mobility. It is concluded here that large-scale sweep structures are capable of generating excessive and persistent force fluctuations which are predominantly responsible for the dynamic process of particle dislodgement. This result supports the impulse criterion as the proper way of accounting for the cumulative effect of coherent structures on particle movement.

Keywords: Initiation of particle motion; Coherent structure; Impulse criterion

\section{Introduction}

Evaluation of the effect of coherent structures on particle mobility remains a key challenge to researchers seeking to formulate models that are capable of accounting for the role of turbulent 
flows on sediment transport under near-threshold conditions [1-4]. Among various approaches,

33 the quadrant analysis has been extensively used to characterize the flow events associated with initiation of particle motion. Specifically, flow processes near sediment particles can be categorized into four velocity quadrant events: (1) $Q_{1 V}\left(u^{\prime}>0, w^{\prime}>0\right)$, (2) $Q_{2 V}\left(u^{\prime}<0, w^{\prime}>\right.$ 0), (3) $Q_{3 V}\left(u^{\prime}<0, w^{\prime}<0\right)$, and (4) $Q_{4 V}\left(u^{\prime}>0, w^{\prime}<0\right)$, where $\left(u^{\prime}, w^{\prime}\right)$ are the streamwise and bed-normal velocity fluctuations, respectively, and the subscript " $V$ " is used for velocity events. An extensive review of the quadrant method was recently provided by Wallace [5].

Particle suspension is frequently attributed to $Q_{2 V}$ (ejection) events due to their pronounced upward velocity [6-8]. Sediment transport as bedload, however, is commonly associated with $Q_{4 V}$ (sweep) or $Q_{1 V}$ (outward-interaction) events because of their particularly strong streamwise velocity [9-12].

A common consideration in previous research has been the importance of instantaneous flow velocity spikes detected around the instant of particle movement [13]. This consideration appears to be logical when initiation of particle motion occurs in a very short time, several tenths of a second [14, 15]. However, recent experimental evidence [16-19] has suggested that, for nearthreshold conditions, only a small fraction $(\sim 10 \%$ for the experiments reported in $[16,17])$ of the velocity spikes or their force realizations that exceed a theoretical critical value can actually dislodge the particle. The duration of velocity spikes has been highlighted because most of the particle dislodgements are attained by peak turbulent events of longer duration. Most of the short-lived spikes can only make the particle vibrate within its sitting bed pocket [16-19]. Here the term "particle dislodgement" is specifically referred to the process of removing a stationary particle permanently from its pocket.

The relevance of turbulence duration motivates this study to consider particle dislodgement as a result of velocity quadrant sequences, rather than a single velocity quadrant event. This perception agrees qualitatively with experimental observations describing the dynamic processes of particle dislodgment driven by coherent structures. For example, Hofland and Booij [20] suggested that a near-bed hairpin-vortex-system (HVS) can initially generate lift forces via small-scale ejections $\left(Q_{2 V}\right)$ to moderately elevate a particle. Subsequently, HVS can exert drag forces via large-scale sweeps $\left(Q_{4 V}\right)$ to dislodge this particle. Similar descriptions of the dislodging processes can be found in other references [15, 21-23]. 
Another challenge in predicting particle dislodgement is the reliability of flow velocity as a proxy for hydrodynamic forces exerted on sediment particles. The pioneering work of Einstein and El-Samni [24] emphasized the major influence of fluctuating pressures rather than velocities on the roughness elements. Direct pressure or force measurements performed on sediment 66 particles have indicated an incomplete coupling between the velocity history and the

67 synchronous pressure/force data record (e.g. [25, 26]). The fluctuations of near-particle velocity 68 do not account fully and uniquely for the corresponding instantaneous fluid forces (e.g. [27, 28]). 69 Researchers have tried to address this rather complicated instantaneous velocity/force correspondence through the use of a time-variant drag coefficient $[9,26]$. The direct measurement of hydrodynamic pressures is therefore considered in this study for a proper 72 examination of fluid forces.

73 The main objective of this paper is to provide a comprehensive perspective on particle 74 dislodgement under a framework of turbulence sequences. Particular emphasis is placed on the 75 periods of particle dislodging processes to underline the cumulative effects of causative 76 turbulence sequences. To achieve this goal, the conventional velocity quadrant analysis is 77 extended to incorporate the quadrant analysis of the associated pressure sequences. Also, a 78 velocity filtering technique is employed here to improve the velocity measurements and, consequently, their coupling with the corresponding pressure records.

80 The rest of the paper is organized as follows. Section 2 presents the experiments used to 81 obtain the velocity and pressure measurements. Section 3 describes the methods of data analysis. 82 The results and interpretations are shown in section 4, a discussion in section 5, and a set of 83 conclusions in section 6.

\section{4}

85

86

87

88

89

\section{Experiment}

The experiment was originally carried out by Celik et al. [9, 25] to evaluate particle mobility under near-threshold conditions. The main aspects of the experiment and related data collection programs are outlined here to keep the paper self-contained. Additional experimental details can be found in the above publications.

\subsection{Physical model}


A $14.4 \mathrm{~m}$ long and $0.6 \mathrm{~m}$ wide water recirculating flume tilted at a slope of $0.25 \%$ was used. The flume test section was covered with two layers of densely-packed single glass beads of diameter $D=12.7 \mathrm{~mm}$. This setting allowed for the full development of turbulent flows under investigation. Table 1 shows pertinent parameters that characterize flow and boundary conditions. The glass-bead boundary was rendered hydraulically rough with a particle (shear) Reynolds number of 424 (defined in Table 1). Also, the ratio of flow depth to particle diameter reported here $(h / D=5.9)$ is typical of the depth-limited flows encountered in gravel-bed rivers [29, 30], enabling meaningful comparisons with field conditions.

For bed mobility considerations, the present experiment was designed for a dimensionless shear stress $(\Theta)$ about one-tenth the critical value obtained from the Shields diagram [31] (Table 1). As a result, extensively mobilized bed condition is not present in this work. However, sporadic dislodgement is possible for highly protruding particles resting on top of the glass-bead layers $[26,32]$. At this stage of very low and highly intermittent bedload transport, the role of energetic near-bed turbulence on particle dislodgement is particularly important [13]. To better understand this role, a simplified arrangement of a single, highly exposed test particle is employed here so that no other protruding particle affects the flow physics upstream of the test particle (Fig. 1). The wakes separated from the upstream base beads are considered as a part of local flow fluctuations.

Table 1 Experimental flow and boundary conditions

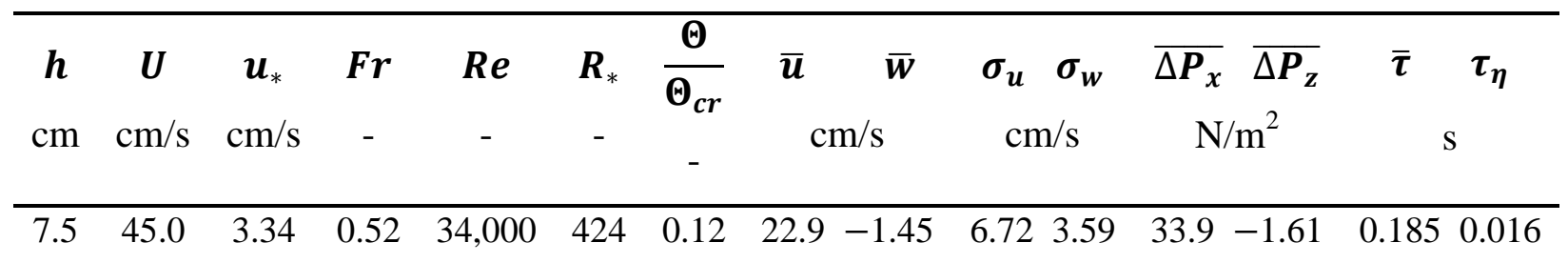

$h=$ flow depth

$U=$ area-averaged flow velocity

$u_{*}=$ bed shear velocity $=\sqrt{\tau_{0} / \rho} ; \tau_{0}=$ mean bed shear stress; $\rho=$ density of water

$F r=$ Froude number $=U / \sqrt{g h}$

$R e=$ flow Reynolds number $=U h / v ; v=$ kinematic viscosity of water

$R_{*}=$ particle (or shear) Reynolds number $=u_{*} D / v ; D=$ particle diameter

$\Theta=$ Shields stress $=\tau_{0} /\left[\rho g\left(S_{G}-1\right) D\right] ; S_{G}=$ specific gravity of particle;

$\Theta_{c r}=$ critical Shields stress $=0.056$ from the Shields diagram

$(\bar{u}, \bar{w})=$ long-term average of the near-particle streamwise and bed-normal flow velocities $(u, w)$ 
$\left(\sigma_{u}, \sigma_{w}\right)=$ standard deviation of $u$ and $w$ respectively

$\left(\overline{\Delta P_{x}}, \overline{\Delta P_{z}}\right)=$ long-term average of the streamwise and bed-normal net pressures $\left(\Delta P_{x}, \Delta P_{z}\right)$

$\bar{\tau}=$ local integral time-scale $=\int_{0}^{\infty} R_{u u}(t-\tau) d \tau ; R_{u u}=$ autocorrelation function of $u ; \tau=$ time shift $\tau_{\eta}=$ local Kolmogorov time-scale $=\sqrt{v / \epsilon} ; \epsilon=$ dissipation rate of local turbulence kinetic energy [33, $34]$

\subsection{Velocity/Pressure measurements}

The near-particle flow velocity and associated hydrodynamic pressures were measured to quantify the influence of coherent structures upon single-particle mobility. At the flume centerline, a $12.7 \mathrm{~mm}$ spherical test particle was permanently placed on top of the two-layer glass beads throughout the experiment. This highly (87\%) exposed test particle was instrumented with four piezoelectric transducers located on the front $\left(P_{1}\right)$, back $\left(P_{2}\right)$, top $\left(P_{3}\right)$ and bottom $\left(P_{4}\right)$ to measure the in-situ instantaneous surface pressures (Fig. 1a). These measurements allowed for the net pressure record in the streamwise $\left(\Delta P_{x}=P_{1}-P_{2}\right)$ and bed-normal $\left(\Delta P_{z}=P_{4}-P_{3}\right)$ directions to be calculated. The drag and lift forces can be approximated by the obtained net pressures (Fig. 1b). A two-component Laser Doppler Velocimetry (LDV) was used to obtain the streamwise $(u)$ and bed-normal $(w)$ velocity records. The location of velocity measurements is along the centerline and one particle diameter upstream of the test particle (Fig. 1a), consistent with similar studies reported in the literature $[15,26]$.

The output signals of simultaneous velocity and pressure measurements were collected via a multichannel signal processor. The pressure transducers operated at a constant sampling rate of $250 \mathrm{~Hz}$. However, the LDV sampling rate varied over time between 250 and $700 \mathrm{~Hz}$, depending on the instantaneous concentration of seeding particles within the measuring control volume. Since the LDV data were nonuniformly sampled, the spline interpolation procedure was used to resample the data at a constant rate of $700 \mathrm{~Hz}$ without overfitting. The resulting uniform LDV data was resampled again at $250 \mathrm{~Hz}$ to obtain a one-to-one synchronized data set of velocity and pressure realizations. Antialiasing filters were used in the above resampling processes.

Compared to the local Kolmogorov time-scale of $0.016 \mathrm{~s}$ (Table 1), the sampling rate of velocity and pressure records $(250 \mathrm{~Hz}$, i.e. time-resolution of $0.004 \mathrm{~s})$ was sufficiently high to resolve boundary turbulence characteristics. The duration of the data collection program for this experiment was $15 \mathrm{~min}$. When compared to the local integral time-scale of $0.185 \mathrm{~s}$ (Table 1), this 
151 period proved to be sufficiently long to secure stationary statistics of the hydrodynamics 152 measurements.
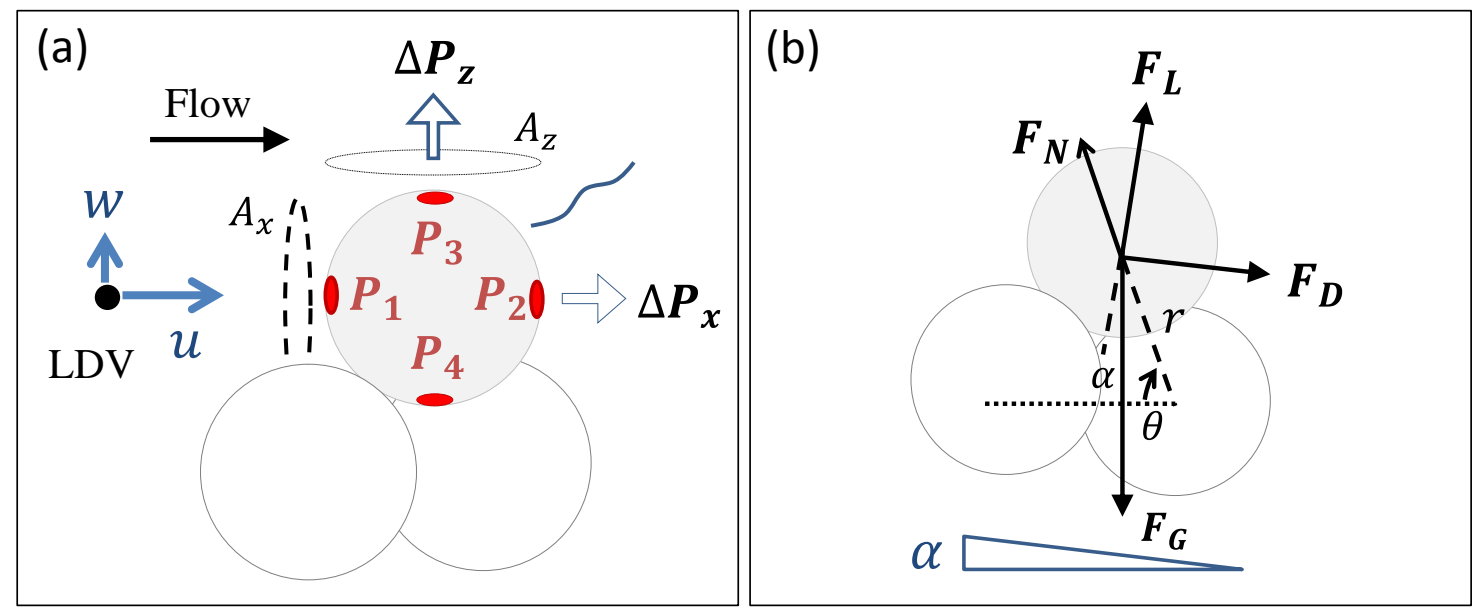

Fig. 1 Schematics of (a) particle arrangement and locations of LDV and pressure transducers $\left(P_{1}\right.$ to $P_{4}$ ), and (b) force diagram of the test sphere. The LDV sampling volume has dimensions of $100 \mu \mathrm{m}$ (streamwise) $\times 100 \mu \mathrm{m}$ (bed-normal) $\times 800 \mu \mathrm{m}$ (spanwise). The $\operatorname{drag}\left(F_{D}\right)$ and lift $\left(F_{L}\right)$ forces are estimated by $F_{D}=\Delta P_{x} A_{x}$ and $F_{L}=\Delta P_{z} A_{z}$, respectively. $A_{x}$ and $A_{z}$ are the projected areas of the spherical particle perpendicular to the streamwise and bed-normal directions, respectively. $F_{G}$ is the submerged particle weight and $F_{N}$ is the contact force. $r$ is the distance between the center of the rolling sphere and the axis of rotation, $\alpha$ is the bed slope, and $\theta$ is the angle between $r$ and the horizontal plane.

\subsection{Time-lag correction}

Despite the synchronization of LDV and pressure transducers, a shift in time is expected among the signals recorded at different locations when responding to a propagating flow event. This type of time-lag is commonly examined via Taylor's “frozen turbulence" hypothesis and an appropriate convective velocity [35]. In contrast to typical considerations of the local timeaveraged flow velocity as the convective speed, more recent research has indicated that the nearbed turbulence would propagate downstream at a speed higher than the local mean flow [35]. It

171 is therefore reasonable to assume that the convective speed is case-specific and that it depends on 172 the dynamics of coherent structures. 
This study considers the conditional average of local flow velocities during the periods of particle dislodgement as the convective speed for the causal coherent structures. The periods of particle dislodgement are identified by a numerical model (section 3.3). The obtained convective speeds can yield the time-lag between the LDV and frontal transducer $P_{1}$, which varies around a mean value of $0.040 \mathrm{~s}$ among different coherent structures. As for the time-lag between $P_{1}$ and the other transducers, the cross-correlation functions of $P_{1}-P_{2}$ and $P_{1}-P_{3}$ signals indicate that $P_{3}$ is behind $P_{1}$ by $0.008 \mathrm{~s}$ and that $P_{2}$ is behind $P_{1}$ by $0.028 \mathrm{~s}$. No noticeable time-lag can be found between $P_{3}$ and $P_{4}$ signals. Correcting the aforementioned time-lag among signals can enhance the reliability of associating rapidly fluctuating turbulence with hydrodynamic impact on the particle.

\subsection{Velocity and pressure coupling}

Fig. 2 shows a representative section of the synchronous velocity and net pressure records used here. The dominant streamwise velocity $u$ and downstream net pressure $\Delta P_{x}$ overall trends are in reasonable agreement. However, a detailed comparison identifies occasional high-frequency velocity fluctuations (superimposed on the major trend) that are not captured in the corresponding net pressure records. These small fluctuations are modulated, or low-pass filtered out, by the test particle and seem to be inconsequential as far as particle mobility is concerned. In this regard, prior experimental evidence has suggested that turbulence structures smaller than the particle size produce no substantial effect on particle mobility [26]. Furthermore, full particle dislodgement is probably accomplished by flow structures as large as at least twice the particle size [14]. Given these indications, it is appropriate to remove the high-frequency velocity signals for revealing the most impactful coherent structures.

Another reason for post-processing the velocity signals is to reduce inconsequential velocity spikes or plunges (red arrows in Fig. 2a). These velocity deviations cause no considerable change in pressure (Fig. 2b), yet leave ample room for misinterpretation of local hydrodynamics due to their prominence among the surrounding velocities. A despiking process is therefore necessary.

The high-frequency velocity fluctuations and inconsequential spikes are removed by appropriate low-pass filters (section 3.1). The consequent improvement is examined by referring to the corresponding net pressure record. Overall, the direct pressure measurements are used to probe the applied hydrodynamic conditions related to particle dislodgement and, moreover, to 
207

208

209

210

211

212

213

214

215

216

217

218

help enhance the veracity of flow physics analysis. This approach is preferable, especially when identifying an appropriate location for obtaining velocity measurements to more accurately determine the fluid forces acting on a sediment particle remains a controversial point [24-26].
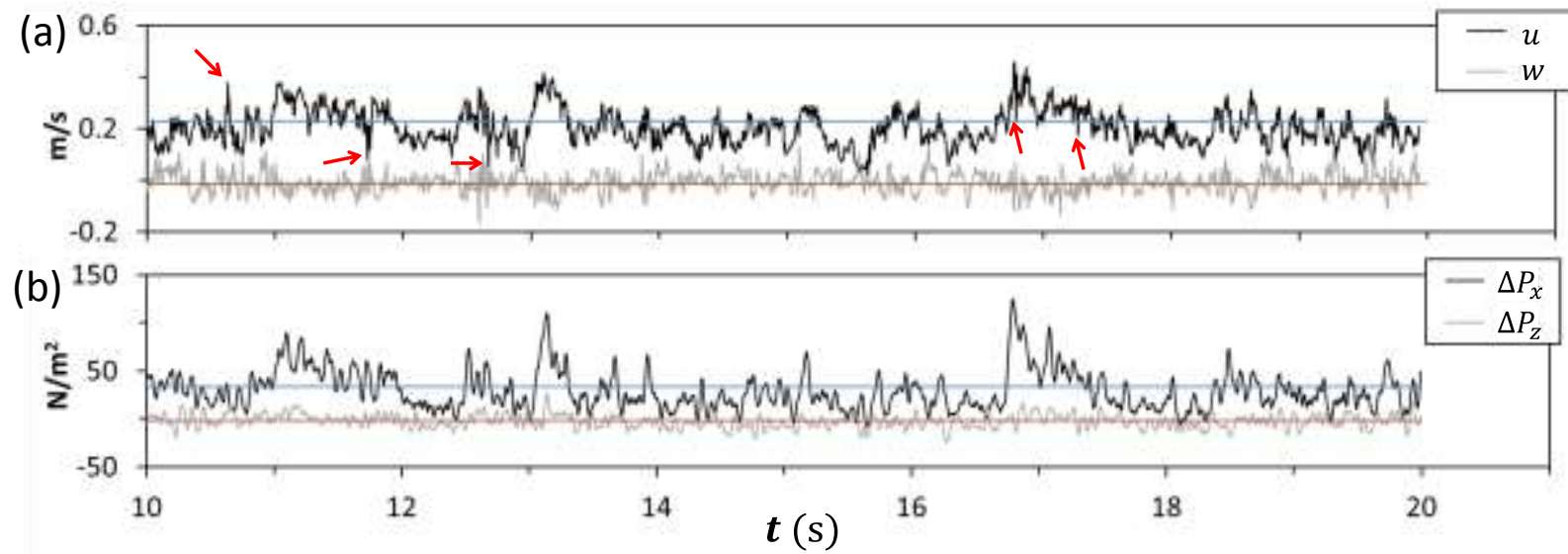

Fig. 2 Representative time series of (a) instantaneous local velocity and (b) corresponding net pressure records. The long-term average values (Table 1) are presented by horizontal lines. The red arrows indicate inconsequential $u$-velocity spikes and plunges that barely have an impact on the net pressure response.

\section{Methods of data analysis}

\subsection{Velocity filtering technique}

In attempts to remove minor turbulence events, velocity filtering techniques usually employ arbitrary criteria. For instance, the threshold "hole size $H$ " used in the conventional quadrant analysis covers a variety of values, typically ranging from 0 to 8 , with even higher values having been reported in the literature [36, 37], where $H$ is defined by $\left|u^{\prime} w^{\prime}\right| \geq H\left(\sigma_{u} \sigma_{w}\right)$. This hole-sizefilter detects the bursting events of particularly large instantaneous Reynolds stresses (- $\left.\rho u^{\prime} w^{\prime}\right)$ [5].

By contrast, the present focus is on the magnitude of the streamwise velocity, a relatively good predictor of particle dislodgement as compared to the instantaneous or time-averaged Reynolds stress $[10,11]$. To remove inconsequential coherent structures that are smaller than the 
225 particle diameter, digital low-pass filters with a cutoff frequency of $f=\bar{u} / D$ are used here to 226 process velocity data. Less attention is focused on the $w$-velocity component due to its 227 insignificant role on dislodgement of a highly protruding particle [9, 25].

228 Fig. 3a exemplifies a section of the post-processed velocity $u_{p p}$ record contrasted with the 229 original $u$ data. The velocities are expressed as pressure predictors for a comparison with the net 230 pressure $\Delta P_{x}$ (Fig. 3b). It is noticed that a striking velocity spike (red arrow) has been attenuated 231 by the filter to a more modest level below the threshold $\Delta P_{x_{c r}}$ for initiation of particle rolling. 232 This spike removal prevents overestimation of hydrodynamic impact because the applied $\Delta P_{x}$ 233 does not exceed $\Delta P_{x_{c r}}$ during the same period of time. Inconsequential small velocity variations 234 that obscure coupling between velocity and pressure are also eliminated. Nevertheless, despite 235 the above improvements, the velocity remains essentially a surrogate measure of hydrodynamic 236 forces (Fig. 3).

237 Among several typical filters such as Chebyshev, Butterworth, and Elliptic, a 6th-order 238 Butterworth filter is chosen for the present experimental setting. It effectively reduces the 239 probability of hydrodynamically inconsequential $u$-velocity spikes by $30 \%$, compared to $22 \%$ 240 reduction by Chebyshev and $25 \%$ by Elliptic. Although the other two filters seem to be able to 241 achieve similar performance, their extra parameters (i.e. ripple factor and selectivity factor) need 242 to be finely tuned to optimize the stopband and passband conditions for particular signals. In 243 order to avoid such arbitrariness, the Butterworth filter is used for general applications. 

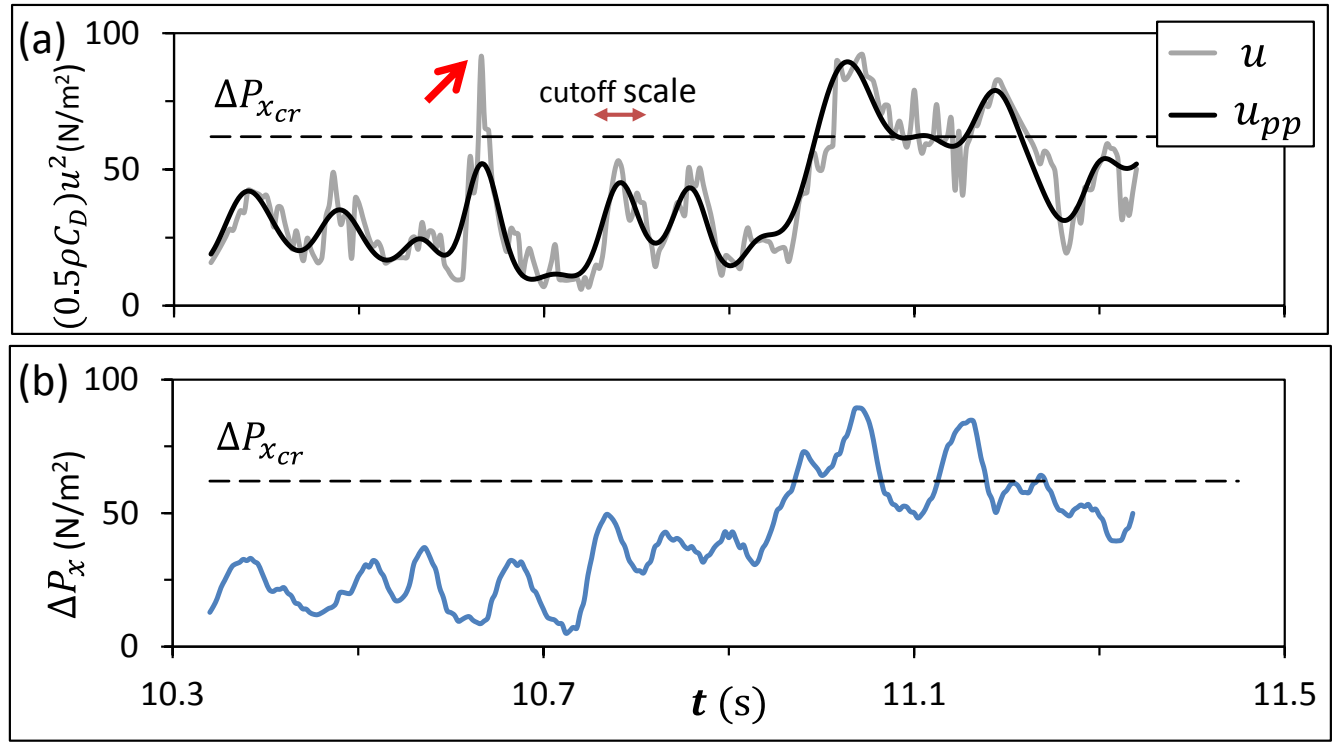

\subsection{Pressure quadrant}

In an effort to enhance our understanding about the time-resolved characteristics of the local flow hydrodynamics and their impact on particle mobility obtained through the traditional velocity quadrant approach, a quadrant-based analysis of the net pressures applied upon the test particle is considered here. Similar to velocity, the net pressure can be categorized into four pressure quadrant events: (1) $Q_{1 P}\left(\Delta P_{x}^{\prime}>0, \Delta P_{z}^{\prime}>0\right)$, (2) $Q_{2 P}\left(\Delta P_{x}^{\prime}<0, \Delta P_{z}^{\prime}>0\right)$, (3) $Q_{3 P}$ $\left(\Delta P_{x}^{\prime}<0, \Delta P_{z}^{\prime}<0\right)$, and (4) $Q_{4 P}\left(\Delta P_{x}^{\prime}>0, \Delta P_{z}^{\prime}<0\right)$, where $\left(\Delta P_{x}^{\prime}, \Delta P_{z}^{\prime}\right)$ are the fluctuations of the streamwise and bed-normal net pressures, respectively, and the subscript " $P$ " is used for pressure events. Fig. 4 illustrates the proposed pressure quadrant plane.

The pressure quadrant approach based on direct pressure measurements can provide an integrated examination of the instantaneous drag and lift forces. For instance, $Q_{1 P}$ reflects a trend 
264 of increasing drag and lift forces when compared to the mean hydrodynamics. It can be regarded 265 as a force fluctuation towards the downstream-upward direction " $\nearrow$ ". On the other hand, $Q_{4 P}$ 266 represents a trend of increasing drag force, but decreasing lift force, which gives a force 267 fluctuation towards the downstream-downward direction " $\searrow$ ". This indication about the direction 268 of force fluctuation is similar to that of velocity quadrants which characterize the direction of 269 velocity fluctuation. Relating the pressure quadrant to the velocity quadrant can give insights 270 about the role of particular flow processes on the instantaneous applied forces.

271 Fig. 4 also shows the thresholds for initiation of particle movement and possible motion 272 modes. The suspension threshold $\Delta P_{z_{c r}}^{\prime}$ (subscript " $c r$ " indicates the critical value) is determined 273 by considering a force balance in the vertical direction: the bed-normal pressure fluctuation $\Delta P_{Z}^{\prime}$ 274 needs to generate sufficient lift force to overcome the submerged particle weight. The rolling 275 threshold $\Delta P_{x_{c r}}^{\prime}$ is calculated by moment balance (Appendix A): the streamwise pressure 276 fluctuation $\Delta P_{x}^{\prime}$ should produce sufficient moment to overcome the resisting moment due to 277 gravity. The threshold $\Delta P_{x_{c r}}^{\prime}$ varies linearly with $\Delta P_{z}^{\prime}$ (Fig. 4) because the bed-normal pressure 278 can influence the apparent particle weight. Particle sliding sometimes accompanies particle 279 rolling if the friction between the particle surface and bed has been overcome. For a situation 280 where $\Delta P_{x}^{\prime}$ is quite large and $\Delta P_{z}^{\prime}$ is close to the suspension threshold, the flow can induce 281 saltation.

282 Need to keep in mind that full particle dislodgement typically takes more than a single 283 quadrant event to be completed. A causal pressure sequence spanning over different quadrant 284 domains is likely to occur under highly fluctuating flow conditions. In such cases, the final 285 motion mode should depend on the cumulative effect of various pressure quadrants. The zones of 286 rolling, saltation, and suspension are specified here only for identifying the pressure 287 requirements in contributing to different motion types. 


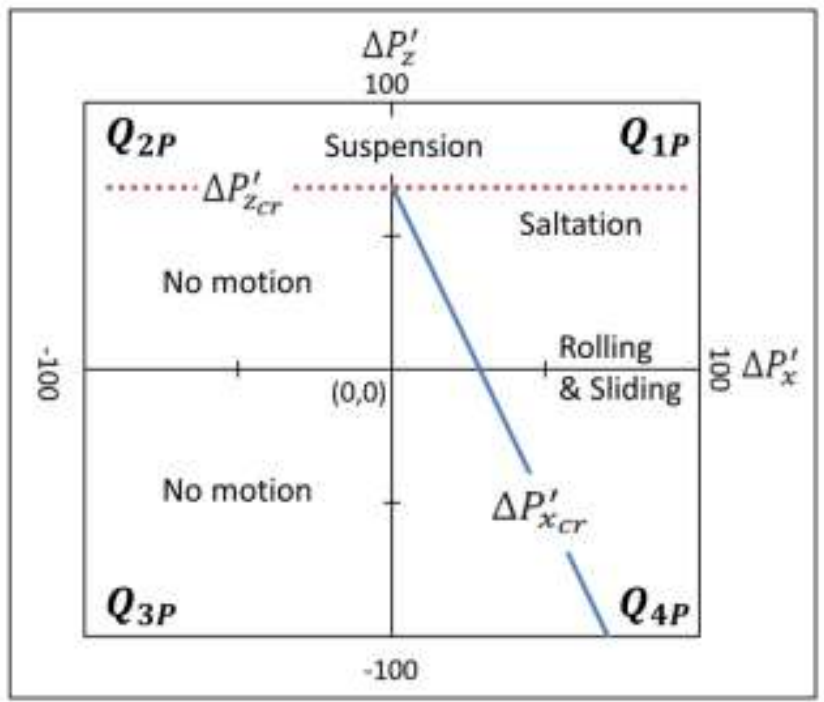

290 Fig. 4 Representative pressure quadrant plane presented in the unit of $\mathrm{N} / \mathrm{m}^{2}$. The origin of the 291 quadrant plane corresponds to the time-averaged net pressures $\left(\overline{\Delta P_{x}}, \overline{\Delta P_{z}}\right)$ (Table 1). The particle 292 rolling and suspension thresholds, $\Delta P_{x_{c r}}^{\prime}$ and $\Delta P_{z_{c r}}^{\prime}$, respectively, are determined for the present 293 particle configuration. $\Delta P_{x_{c r}}^{\prime}$ is marked by a solid-blue line and $\Delta P_{z_{c r}}^{\prime}$ is marked by a dashed-red 294 line.

\subsection{Simulating particle dislodgement}

297 A trade-off of the direct pressure measurement is that the instrumented particle needs to be held 298 in place throughout the experiment $[22,27]$. Identifying particle movement, hence, relies on a 299 numerical simulation. This study uses a perfect-rolling model, reported by Schmeeckle and 300 Nelson [38], to predict particle dislodgement (Appendix A). Specifically, the angular 301 displacement of the particle is calculated by considering the instantaneous moment induced by 302 fluid forces that can be approximated by the net pressure measurements (Fig. 1b). The rolling 303 trajectory is established over time until reaching the topmost of the bed pocket. Selection of the 304 rolling mode is warranted because of the results obtained during mobile-particle experiments conducted by Celik et al. [16] under nearly identical hydrodynamic conditions.

Although this numerical approach has already been used to predict incipient rolling [19, 3840], the particular advantage here is access to the directly measured net pressures. This allows 
for more accurate detection of excessive fluid forces that surpass the rolling threshold, as contrasted to the force predictions made by nearby velocity measurements [38] or by theoretical approximation based on the law of the wall [40].

A total of 105 particle dislodgements are identified throughout the 15-min data record via the perfect-rolling simulation. This result is in very close agreement with the 104 dislodgements detected by Celik et al. [16] over a 15-min period in the mobile particle experiment, which constitutes a replicate of the fixed particle one. This agreement provides a great deal of confidence regarding the use of velocity/pressure data obtained from the static case to describe the general particle dislodgement characteristics. However, the detailed velocity/pressure conditions for a static and a mobile particle are not expected to be the same during the dislodgement process. This is not a serious limitation considering that each particle dislodgement is different, controlled by a unique combination of pressure and velocity conditions. A representative case of the rolling simulation is detailed in section 3.4 to emphasize the dynamic process of particle dislodgement.

\subsection{Time-resolved rolling trajectory}

The use of continuous simulation enables the calculation of a time-resolved rolling trajectory and underlines the dynamic nature of particle dislodgement. Fig. 5 exemplifies a simulated dislodgement accomplished by several turbulence sequences. The hydrodynamic impact is reflected in the dominant net pressure fluctuation $\Delta P_{x}^{\prime}$. The bed-normal fluctuation $\Delta P_{z}^{\prime}$ is factored into the critical value $\Delta P_{x_{c r}}^{\prime}$ by allowing a time-variant threshold value (Fig. 5a). Specifically, an upward-directed pressure fluctuation $\left(\Delta P_{z}^{\prime}>0\right)$ lowers $\Delta P_{x_{c r}}^{\prime}$ by decreasing the submerged particle weight and vice versa. The particle location is represented by $\varphi$, the ratio of angular displacement to the angle of repose (Fig. 5b). A negative $\varphi$ indicates a location upstream of the resting pocket.

In Fig. 5 an incomplete particle dislodgement is launched by the first excess pressure sequence (labeled as "A"), leading to reverse rolling towards the bed pocket (decreasing $\varphi$ ) and subsequent vibration within the pocket. The vibration is characterized by a very short period of slightly negative $\varphi$ (red arrow). Another failed attempt of particle dislodgement " $\mathrm{B}$ " is captured at a later point in time, but it barely reveals within-pocket vibration. Two consecutive excess 
pressure sequences, " $\mathrm{C}_{1}$ " and " $\mathrm{C}_{2}$ ", cause continuous rolling towards downstream and eventually 338 complete the particle dislodgement process.

During the dislodging process "C", a significant drop in $\Delta P_{x_{c r}}^{\prime}$ results mostly from the fast reducing particle resistance $F_{g} \cos (\theta)$ as $\theta$ changes with particle location. The angle $\theta$ increases 341 from $0.4 \pi$ at the original resting site to nearly $\pi / 2$ at the topmost location (Fig. 1b), where the 342 counteracting moment resisting particle rolling becomes almost zero. Consequently, the greatly 343 lowered threshold facilitates forward particle movement for the excess sequence ' $\mathrm{C}_{2}$ ', even 344 though " $\mathrm{C}_{2}$ " is less energetic than the preceding sequence " $\mathrm{C}_{1}$ ". Based on this dynamic 345 dislodgement scenario, a time-sequence approach is well suited for the proper consideration of 346 the successive destabilizing turbulence events.

347

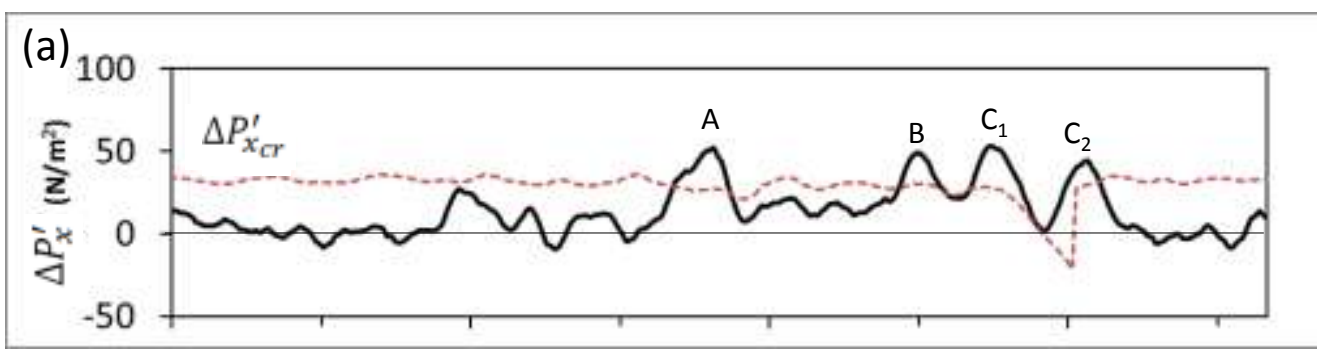

(b) $1.4 \quad \varphi=1$, complete dislodgement

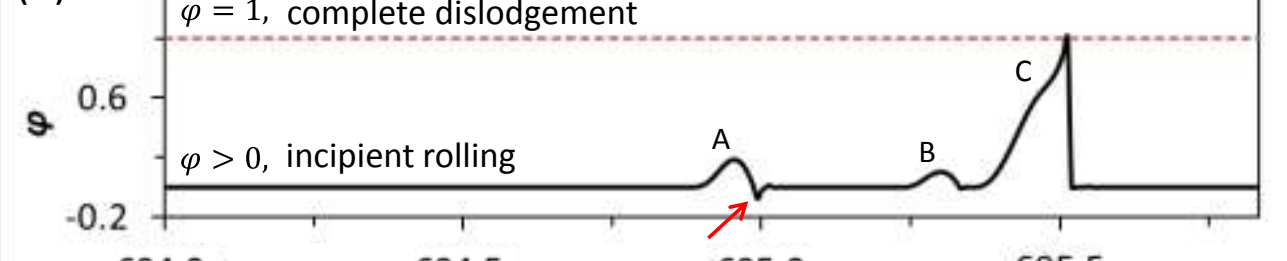
694.0 694.5 $\boldsymbol{t}(\mathrm{s}) \quad 695.0$ 695.5

349 Fig. 5 Representative time series of (a) net pressure fluctuation $\Delta P_{x}^{\prime}$ and (b) resulting rolling 350 progress expressed by the ratio $\varphi$ of angular displacement to the angle of repose. The rolling 351 threshold $\Delta P_{x_{c r}}^{\prime}$ varies with $\Delta P_{z}^{\prime}$ over time and drops significantly when the particle is moderately 352 displaced. The red arrow indicates a short period of vibration after an incomplete dislodgement.

\subsection{Ensemble-average analysis}


In addition to the essential moving fluid parcels that directly impart momentum on particles (e.g. excess pressure sequences shown in Fig. 5b), the prevalent characteristics of the related largescale coherent structures are examined here. This is pursued via an ensemble-average analysis performed over the entire 105 particle dislodgement cases. The period of ensemble-averaging processes is selected as $3.0 \mathrm{~s}$, more than 15 times the integral time-scale of $0.185 \mathrm{~s}$, to account for the effects of macro-turbulence. The number of dislodgement replicates considered here is sufficiently large to secure statistically stationary results (Appendix B).

\subsection{Quadrant probability}

The temporal records of velocity and pressure quadrants are of particular interest. The most frequently encountered quadrant events are highlighted throughout the ensemble-averaged time series to characterize coherent structures. For comparison purposes, the probability $F_{i}(t)$ of finding the flow at quadrant $i$ at a given time $t$ can be defined by

$F_{i}(t)=\frac{n_{i}(t)}{\sum_{j=1}^{4} n_{j}(t)} ;$ for $i=1,2,3,4$,

where $n_{i}$ is the number of $i$-th quadrant realizations.

\section{Results}

The velocity and pressure (quadrant) sequences associated with particle dislodgement are presented here to verify the proposed turbulence sequence approach. The overall statistics of causative quadrant events, including the ensemble-averaged time series, provides characteristics of relevant coherent structures. For presentation purposes, a new time scale $t_{E}$ is used here so that different dislodgement cases can be compared easily by designating the instant of initiation of particle movement at $t_{E}=0$. Particle dislodgement is completed at $t_{E}=T_{D}$, with $T_{D}$ the duration of the dislodging process.

\subsection{Case studies}

Two different dislodgement cases are considered here (Fig. 6). Case 1 is characterized by a major velocity lobe having high magnitude and short duration (Fig. 6a). Case 2 exhibits the opposite trend (Fig. 6b), where the fluid parcel is less energetic but relatively extended as compared to Case 1. Also, Case 2 shows abundant high-frequency velocity fluctuations, 
382 indicating a highly unsteady condition which likely stems from the interactions between coherent

383 structures and background turbulence. The velocity filter is quite useful for removing 384 inconsequential events under such unsteady conditions (blue arrows). In both cases, the post385 processed velocities are in better agreement with the synchronous pressures (Fig. 6 insets), 386 compared to the original velocity records.

387 As expected, velocity quadrant signatures are subjected to some minor changes after 388 performing the filtering process. For the entire 15-min measurement period $(225,000$ 389 realizations), the maximum change in probability (or fractions) among all four quadrant 390 categories is $<2 \%$. However, a somewhat considerable quadrant variation can be noted at a 391 smaller time-scale such as the dislodgement duration $T_{D}$. For example, Case 2 has a probability 392 change as high as $11 \%$ in favor of $Q_{4 V}$ (Fig. 6d). The highly unsteady flow of Case 2 appears to 393 be sensitive to velocity filtering, contrasted to Case 1 with a minor $2 \%$ change (Fig. 6c). In 394 addition to the probability, the magnitude of velocity quadrants is modified to a more modest 395 level.

Case 1
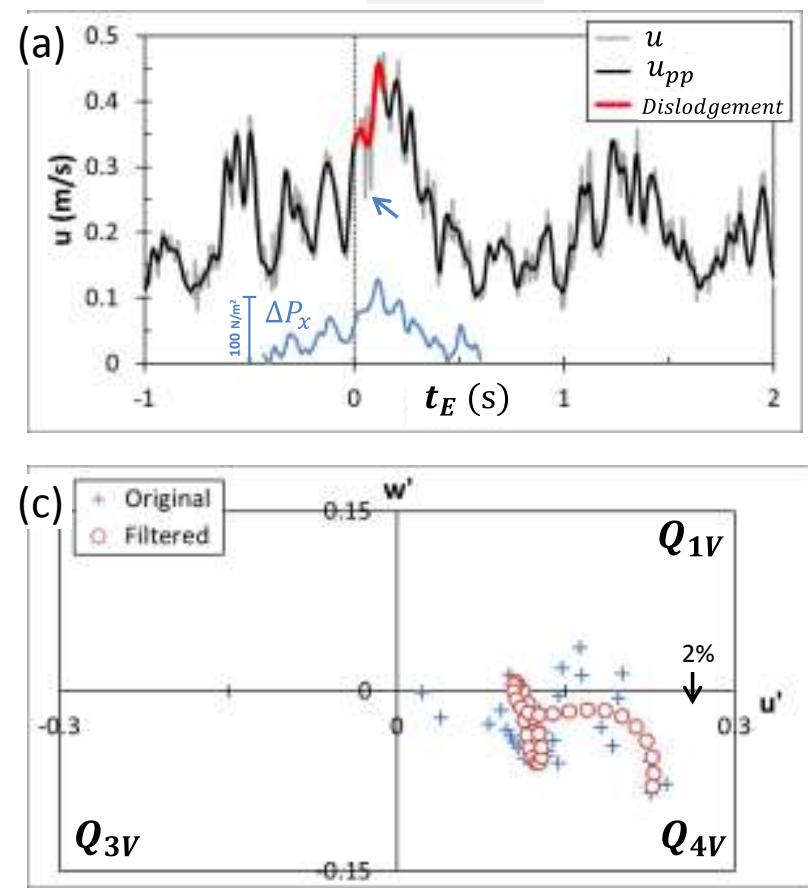

\section{Case 2}
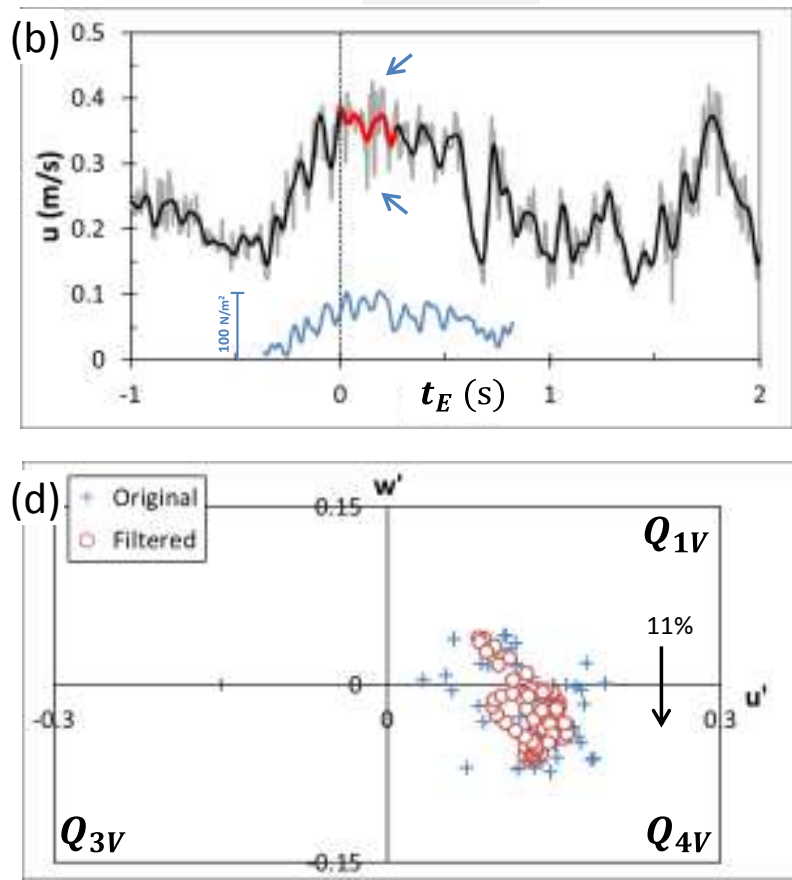

Fig. 6 Temporal records of original velocity $u$ and post-processed velocity $u_{p p}$ for (a) Case 1 and (b) Case 2. The velocity traces during the period of particle dislodgement are highlighted by 
400

401

402

403

404

405

406

407

408

409

410

411

412

413

414

415

416

417

418

419

420

421

422

423

424

425

426

427

red lines, and their quadrant signatures are presented in (c) and (d) for Cases 1 and 2, respectively. The corresponding $\Delta P_{x}$ traces are plotted in the insets of (a) and (b) for comparison purposes. The scale right next to the $\Delta P_{x}$ traces represents a magnitude of $100 \mathrm{~N} / \mathrm{m}^{2}$.

\subsection{Sequential velocity quadrants}

In Cases 1 and 2 the $Q_{4 V}$ and $Q_{1 V}$ events related to particle dislodgement are shown in Figs. 6c and $6 \mathrm{~d}$. Their sequence characteristics are further demonstrated in Fig. 7 to emphasize the timevariant nature of the causal turbulence. A complete dislodgement takes the full length of the quadrant sequences exhibited therein to be achieved. To improve clarity, quadrant events are marked differently based on their association with the first $\left(t_{E}=0 \sim 0.5 T_{D}\right)$ or second half $\left(t_{E}=\right.$ $0.5 T_{D} \sim T_{D}$ ) of the dislodgement periods. The trace (or direction) of quadrant transitions is indicated by single-arrow lines.

Generally, the original velocity quadrants display erratic transitions between successive events (Figs. 7a and 7b). Although a very rough trend can be identified, the convoluted quadrant traces make the flow physics difficult to interpret meaningfully. The impactful flow processes are obscured by such unsteadiness due to the interactions with local turbulence, particularly for Case 2 (Fig. 7b).

The velocity filter effectively improves the velocity quadrant sequences by removing outliers and provides smoother transitions between successive points (Figs. 7c and 7d). In Fig. 7c the filtered quadrant trace clearly exhibits a short period of intensifying downward velocity fluctuations (temporarily enlarged negative $w^{\prime}$ ) in the beginning and then a strengthening flow in the direction of "У". This trend indicates a progressively advancing coherent structure that is characterized mainly by strong sweeps $\left(Q_{4 V}\right)$. The brief appearance of outward-interactions $\left(Q_{1 V}\right)$ supports the above statement. According to Hofland et al. [27] using Particle Image Velocimetry (PIV) mapping techniques, $Q_{1 V}$ appears briefly near a protruding particle right before the flow field is dominated by $Q_{4 V}$ of the coherent structure.

The more complex local flows of Case 2 can be regarded as a generalized version of Case 1. In Fig. $7 d$ the entire quadrant trace consists of several intensifying-weakening cycles regarding 
$428 u^{\prime}$ magnitude, suggesting a series of distinct fluid parcels flowing past the test particle. As a 429 result, the particle experiences consecutive hydrodynamic impacts of highly variable magnitude.

Case 1

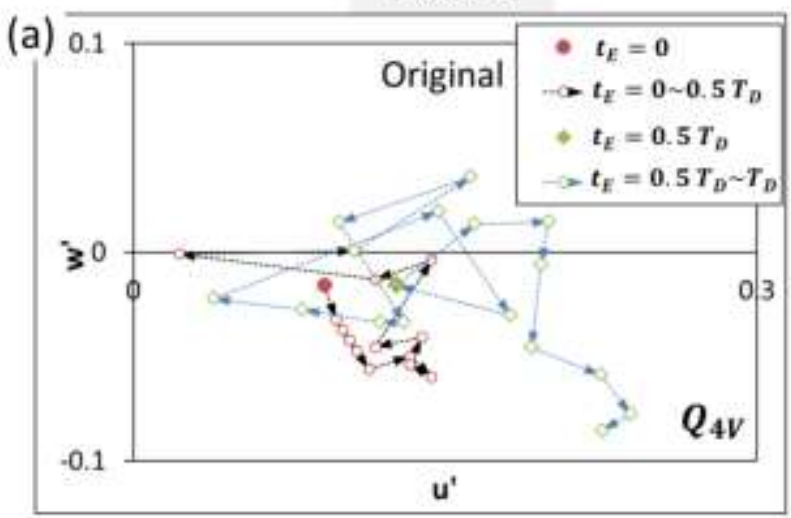

(c)

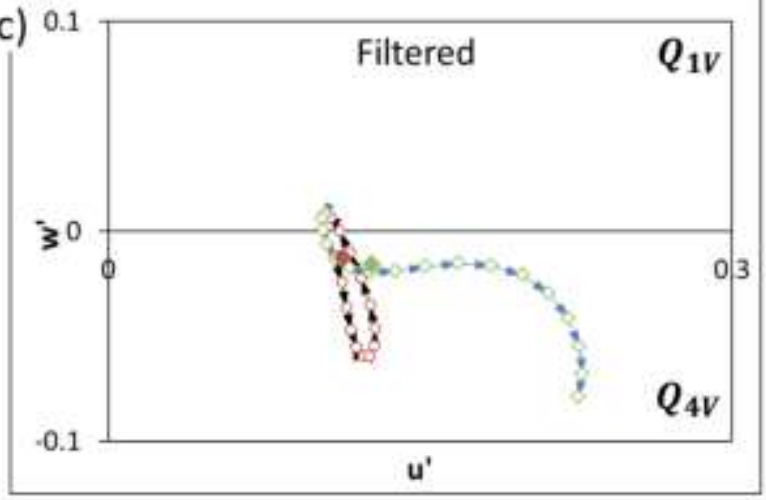

(e)

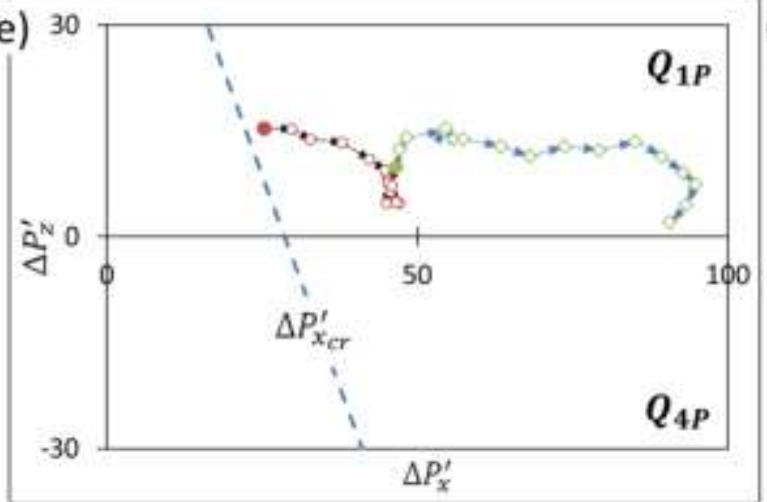

Case 2
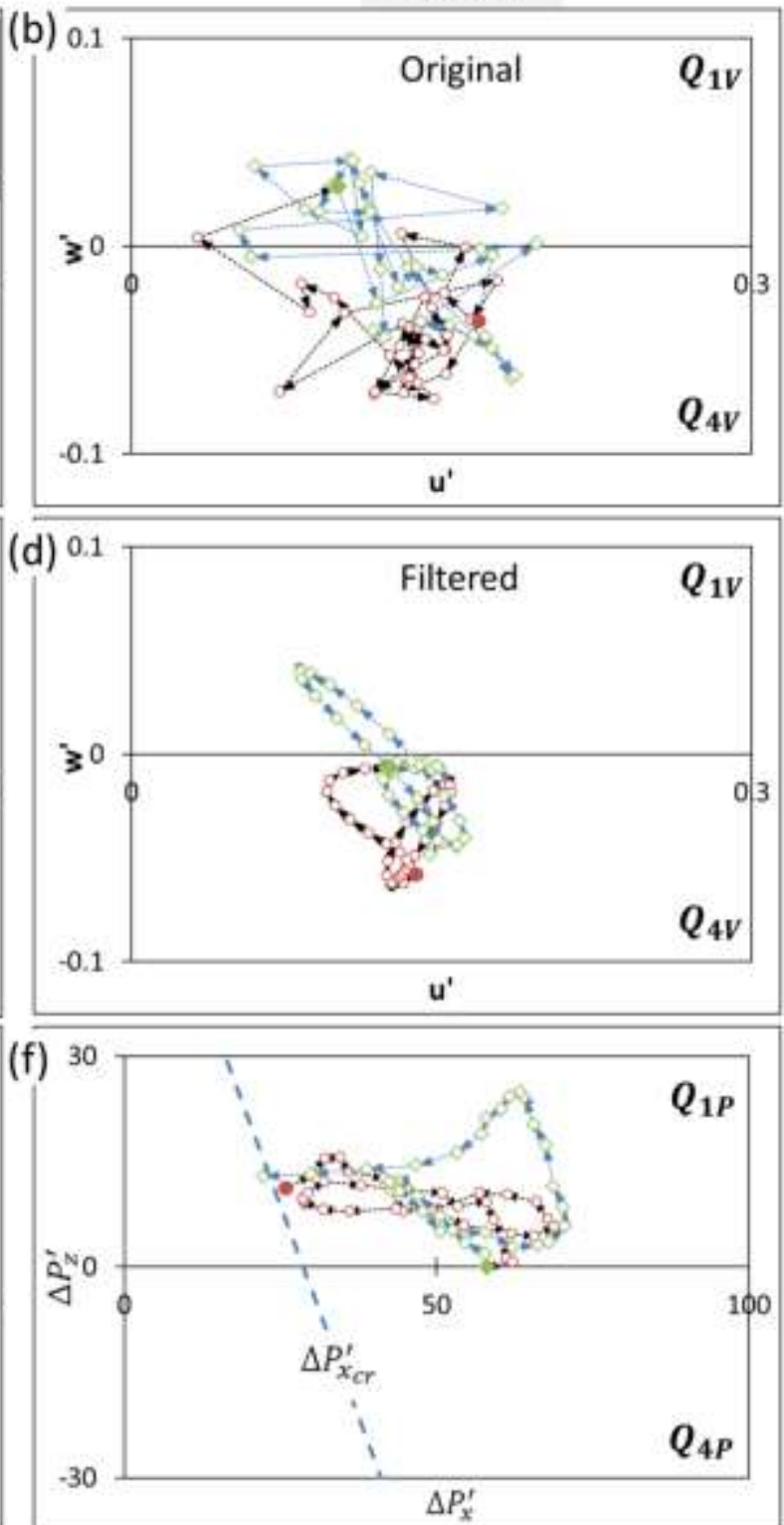

432 Fig. 7 Quadrant sequences of Cases 1 and 2 respectively: (a) and (b) present the original velocity 433 quadrant traces; (c) and (d) present the post-processed velocity quadrant traces; (e) and (f) show 434 the causal pressure quadrant traces. The blue broken lines in (e) and (f) represent the critical 435 pressure fluctuation $\Delta P_{x_{c r}}^{\prime}$ for initiation of particle rolling. 


\subsection{Sequential pressure quadrants}

438

439

440

441

442

443

444

445

446

447

448

449

450

451

452

453

454

455

456

457

458

459

460

461

462

463

464
The hydrodynamic impact of Cases 1 and 2 is presented more conclusively by pressure quadrant sequences than by velocity quadrant sequences (Figs. 7e and 7f). In the rolling process, the test particle experiences mostly the pressure fluctuations characterized by $Q_{1 P}$, indicating a trend of increasing drag and lift forces (“ $\nearrow ”)$. The resultant force either almost monotonically increases (Fig. 7e) or somewhat regularly fluctuates over time (Fig. 7f), based on the complexity of the approaching coherent structures. The force variations can also be perceived via the dominant $\Delta P_{x}$ contribution shown in the insets of Figs. $6 a$ and $6 b$.

The temporal patters of pressure quadrant sequences are more congruent with the postprocessed velocity quadrant sequences than with the original ones (Fig. $7 \mathrm{c} \rightarrow 7 \mathrm{e}$ and Fig. $7 \mathrm{~d} \rightarrow$ 7f). Even so, the improved velocity quadrant realizations cannot reflect particle mobility completely due to the following two reasons.

Firstly, it is not easy to define a unique threshold velocity quadrant that can correspond to the critical pressure quadrant $\left(\Delta P_{x_{c r}}^{\prime}\right)$. The very first event of pressure quadrant sequences is close to the deterministic threshold $\Delta P_{x_{c r}}^{\prime}$ (red circles in Figs. 7e and 7f), whereas the first velocity quadrant event varies considerably between Cases 1 and 2 (Figs. 7c and 7d). The varying incipient velocity quadrant highlights the difficulty of identifying a single characteristic velocity to predict particle motion. Therefore, the traditional incipient velocity method is not suitable for the near-threshold conditions considered here.

Secondly, velocity fluctuations are not entirely consistent with pressure fluctuations regarding both the resultant direction and magnitude. The upward pressure fluctuations $\left(Q_{1 P}\right)$ are associated with not only the upward velocity fluctuations $\left(Q_{1 V}\right)$ but also the downward velocity fluctuations $\left(Q_{4 V}\right)$. Also, the increasing/decreasing magnitude of pressure quadrants is not closely reflected by the trend of velocity quadrants (e.g. $t_{E}=0 \sim 0.5 T_{D}$ in Fig. 6 c compared with Fig. 6e). As a result, the approaches based on velocity fluctuations in estimating particle mobility, such as Reynolds stress methods, can be compromised by such velocity-pressure incongruence.

Overall, pressure quadrant sequences can accurately characterize the consecutively applied fluid forces, while velocity quadrant sequences can reveal the relevant flow processes. Used in 
tandem, these quadrant types can provide a better understanding about cause and effect relation between hydrodynamic processes, including coherent structures, and particle movement.

\subsection{Statistics of causal quadrant sequences}

As shown in Fig. 7, the period of residence in individual quadrant domains changes considerably throughout the entire quadrant sequences and among different cases. Fig. 8 provides visualization of the associated temporal fractions to demonstrate the domain transitions. Cases 1 and 2, along with three other dislodgement cases (3-5), are exemplified here.

In Fig. 8a the velocity quadrant sequences of Cases 1 to 4 can all be categorized as the same sequence type of $\left\{Q_{4 V}, Q_{1 V}, Q_{4 V}\right\}$, solely based on the order of appearance of the quadrant types. Similarly, in Case 5 the flow processes can be characterized by the sequence of $\left\{Q_{4 V}, Q_{1 V}\right\}$. This simplified characterization is mainly intended to capture the overall process qualitatively, thus ignoring that the temporal fraction related to each quadrant domain is quite different. The duration aspect of quadrant sequences remains important as far as particle dislodgement is concerned.

In Fig. $8 \mathrm{~b}$ the pressure quadrant sequences are categorized in the same manner. Furthermore, very short residence in a quadrant domain $\left(<5 \%\right.$ of $\left.T_{D}\right)$ is neglected to highlight the most impactful quadrant groups. For instance, $Q_{4 P}$ in Cases 2 and 3 (red arrows) is negligible. As a result, the consecutive hydrodynamic impact of Cases 1 and 2 is characterized by a pressure quadrant sequence residing exclusively within $\left\{Q_{1 P}\right\}$, and that of Cases 3 to 5 is reflected by $\left\{Q_{1 P}, Q_{4 P}, Q_{1 P}\right\}$.

Examining the entire 105 dislodgement cases under the above method yields the overall occurrence probability of the causal quadrant sequences (Fig. 9). Fig. 9a shows that most of particle dislodgements $(\sim 80 \%)$ are completed during the equally frequent flow sequences of $\left\{Q_{4 V}, Q_{1 V}, Q_{4 V}\right\}$ and $\left\{Q_{1 V}, Q_{4 V}\right\}$. The causative hydrodynamics appears sequentially as $\left\{Q_{1 P}, Q_{4 P}, Q_{1 P}\right\}$ or exhibits consistent $\left\{Q_{1 P}\right\}$ all the time. These characteristic quadrant sequences are more representative of the particle dislodging process than the results obtained through the conventional single quadrant method. 

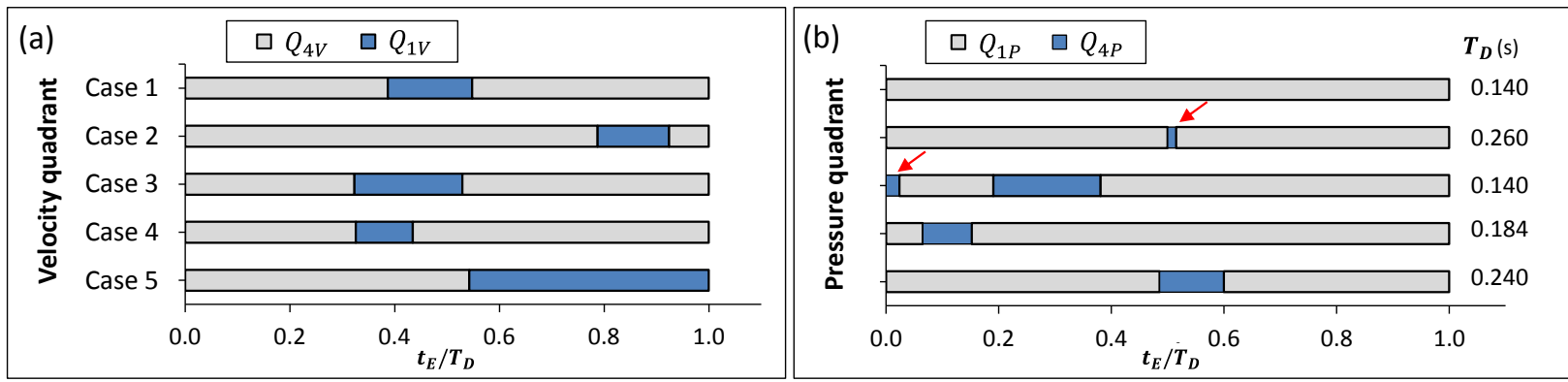

Fig. 8 Visualization of residence time in different (a) velocity quadrant domains and (b) pressure quadrant domains. The residence time is normalized by the dislodgement duration, which is specified in (b) for individual cases.

498
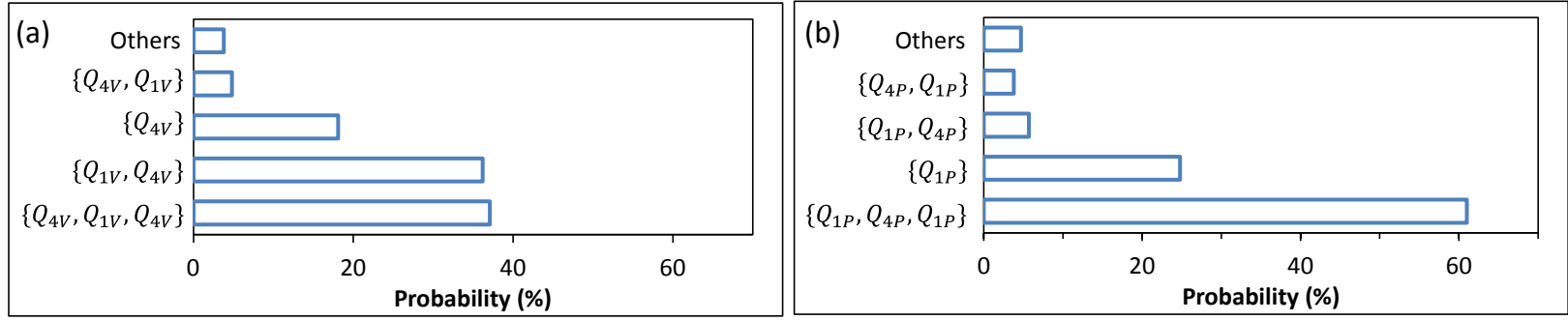

Fig. 9 The occurrence probability of various sequence types: (a) velocity quadrant sequences and

501 (b) pressure quadrant sequences

\subsection{Ensemble-averaged temporal records}

504 So far the essential hydrodynamic conditions that directly cause particle dislodgement have been described comprehensively. A broader perspective on the relevant coherent structures is then presented via the $3.0 \mathrm{~s}$ temporal records of ensemble-averaged variables. The resultant velocity, pressure, and corresponding probability of their quadrant signatures are of particular interest (Fig. 508 10). In this ensemble averaging process, the time interval considered for grouping data is $0.04 \mathrm{~s}$ 509 (Appendix B), accounting for 10 data readings from each registered dislodgement. Therefore, 510 each ensemble-averaged value lumps a total of 1050 velocity/pressure readings.

511 In Fig. 10, the characteristic quantities exhibit an asymmetrical, large-scale pattern spanning 512 roughly over $2.0 \mathrm{~s}\left(t_{E}=-0.5 \sim 1.5 \mathrm{~s}\right)$. This flow pattern (structure) is characterized by abruptly 513 increasing velocity and pressure signals (Figs. 10a and 10b), which exceed the threshold of 
514 incipient rolling conditions at $t_{E}=0 \mathrm{~s}$ and reach their peak values at $t_{E} \approx 0.1 \mathrm{~s}$. These signals

515 last for sufficiently long time to complete the dislodgement. Succeeding the particularly strong

516 spike is a weak, yet long-lasting, wake that smoothly approaches the long-term average level.

517 During this flow process, the dominant velocity quadrant $Q_{4 V}$ and pressure quadrant $Q_{1 P}$ (Figs.

$51810 \mathrm{c}$ and 10d) bear strong resemblance to those documented in the velocity and pressure traces

519 (Figs. 10a and 10b). It is worth mentioning that all of the 105 dislodgements were completed at a

520 later time, $0.128 \mathrm{~s} \leq T_{D} \leq 0.480 \mathrm{~s}$, than the instant the peak velocity and net pressure values

521 occurred (see Fig. 10).

522 Overall, the ensemble-averaged records indicate an evident coherent structure deviating from 523 the mean flow. This $Q_{4 V}$ dominant structure very likely belongs to the lower portion of near-bed 524 span-wise vortices that have been extensively identified by PIV and similar visualization 525 techniques $[22,41,42]$. When this $Q_{4 V}$ structure appears near the sediment particle, the applied 526 net pressures (corresponding to quadrant $Q_{1 P}$ ) increase the probability of particle dislodgement.

527
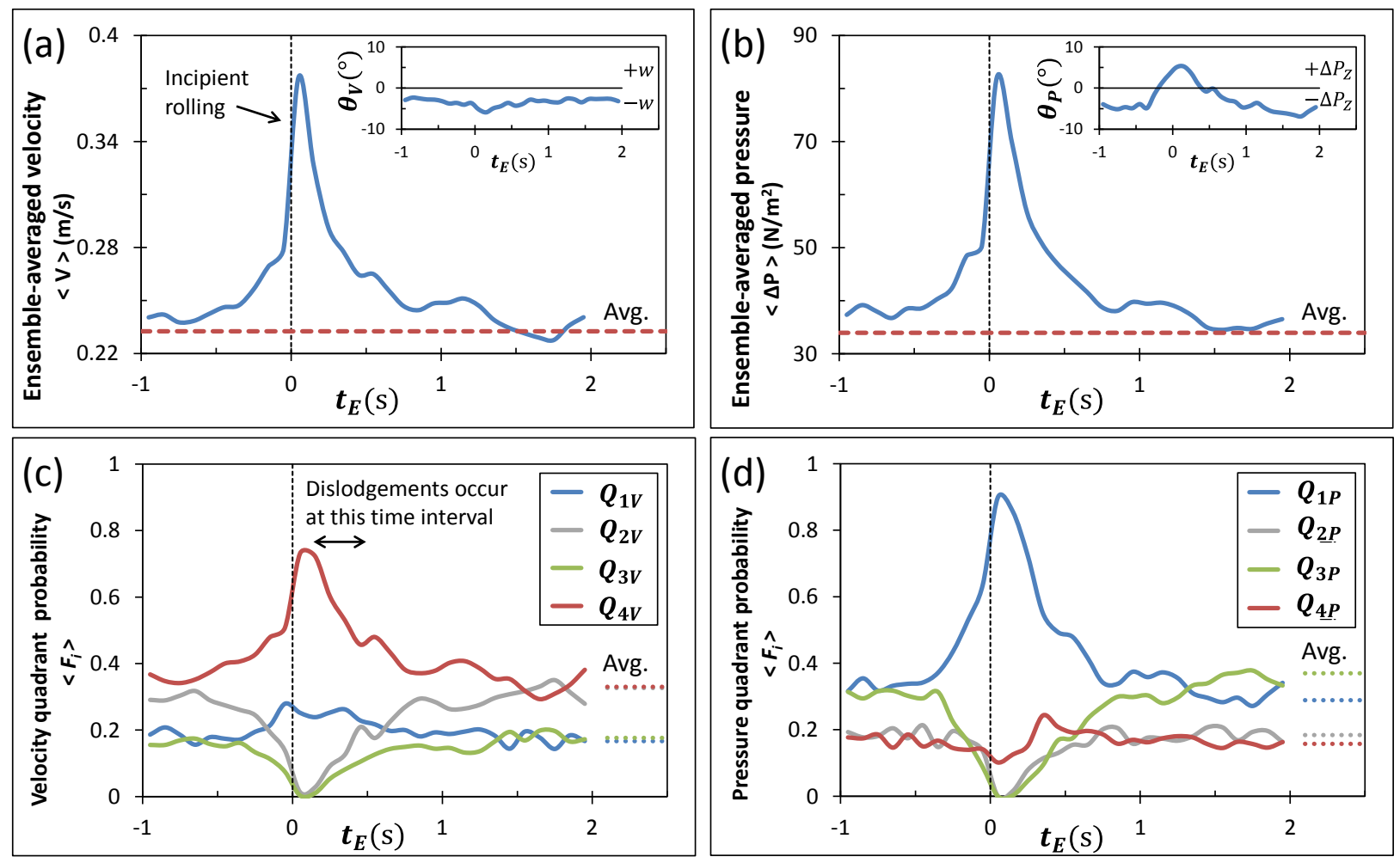

Fig. 10 Ensemble averaged traces: (a) the resultant velocity $V=\left(u^{2}+w^{2}\right)^{0.5}$ with corresponding angle $\theta_{V}=\tan ^{-1}(w / u)$, (b) the resultant net pressure $\Delta P=\left(\Delta P_{x}^{2}+\Delta P_{z}^{2}\right)^{0.5}$

531 with corresponding angle $\theta_{P}=\tan ^{-1}\left(\Delta P_{z} / \Delta P_{x}\right)$, and (c) and (d) the probability of velocity and 
532 pressure quadrants, respectively. The long-term average quantities are presented by horizontal 533 lines.

\section{Discussion}

For more than a century significant efforts have been made to clearly identify and quantitatively determine the critical conditions for particle movement. The temporally and spatially averaged flow parameters have been widely used for engineering applications [43]. Subsequently, a better understanding of turbulent flows allowed for a time-resolved analysis of flow physics near threshold conditions. Considering the random and intermittent nature of sediment movement that prevails near-threshold conditions, it is reasonable, as a first step, to highlight the importance of the extreme instantaneous quantities in highly fluctuating flow fields. The probabilistic properties of peak instantaneous forces or their velocity equivalents are of particular interest.

However, the peak magnitude of fluid forces alone is inadequate to capture the full process of particle dislodgement (e.g. Fig. 5a). From an energy perspective, the applied force needs to last for a sufficiently long period of time in order to perform the required mechanical work to move the particle for a certain distance. To account for the relevance of force duration, Diplas et al. [17, 44] proposed using hydrodynamic impulse (force magnitude times its duration) as a more comprehensive criterion to predict particle dislodgement. The dislodgement attributed to the instantaneous peak force, in fact, can be regarded as a special case of impulse concept, where an extremely large force only requires a very short period of time to impart the necessary momentum for particle dislodgement. For near-threshold conditions, such scenarios, though physically possible, are expected to be very rare. A modest force scenario, moderately exceeding the initiation of motion criterion, requires a longer duration to convey the same impulse as that conveyed by the extremely large force. The latter case is expected to be more representative of the flow events resulting in particle dislodging processes. As it has already been pointed out in recent studies, given the highly unsteady nature of the hydrodynamic forces, it is more appropriate to characterize particle threshold conditions via the concept of minimum impulse or energy rather than minimum force $[9,14,17,25]$. 
Fig. 11 shows the rate of impulse transfer made by two different turbulent processes mentioned previously. The applied impulse is computed by multiplying the varying resultant

562 force by its corresponding duration. In Fig. 11 the variable $\beta$ indicates the ratio of the cumulative 563 impulse at any given $t_{E}$ to the total impulse amount required for particle dislodgement $(\beta=1)$.

564 The slope of $\beta$ curves reflects how fast the flow can impart momentum onto the particle via 565 impulse and thus how quickly it can dislodge it. As expected, the stronger turbulent flow event of 566 Case 1 attains dislodgement $(\beta=1)$ more quickly compared to the less energetic flow of Case 2. 567 This result confirms the veracity of impulse as a consistent criterion to predict particle 568 dislodgement even when subjected to different hydrodynamics.

569 Fig. 11 also reveals different stages in the dislodging process based on the slope change. Case 5701 has a mild slope initially and a rapidly increasing slope in the later stages. The rapidly 571 increasing slope results from the combined effect of the surge in force magnitude (Fig. 6a inset) 572 and coincident decrease in the threshold value (similar to that shown in Fig. 5a, event "C"), thus 573 boosting momentum transfer significantly. This case underlines an accelerating process of 574 dislodgement once the particle has been partially mobilized. In the sense of threshold of motion, 575 the initial phase of fluid force magnitude relative to particle resistive forces can be most critical 576 in determining particle mobility. Such considerations may explain why many researchers are 577 inclined to regard particle dislodgement as a matter of force magnitude or moment balance of the 578 instantaneously applied forces [45], and refer to the peak forces as the sole cause of particle 579 dislodgement. On the contrary, Case 2 shows that the consecutive hydrodynamic impact, 580 including the peak events, contribute to the overall development of particle dislodgement (Fig. 581 11). This process should be better characterized by the turbulence sequences and their respective 582 durations than by the single instantaneous quantities. 


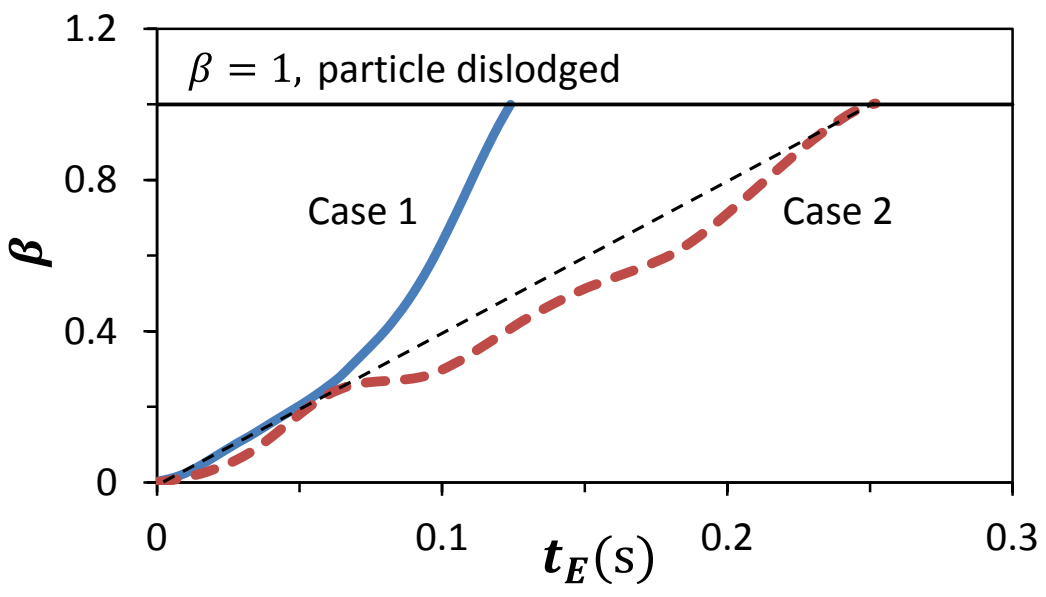

Fig. 11 The rate of impulse transfer associated with turbulent processes of Cases 1 and 2 .

\section{Conclusions}

The overarching goal of this study is to gain a better understanding of the pertinent hydrodynamic processes leading to particle dislodgement under near threshold of motion conditions. To accomplish this objective, this study demonstrates the utility of the following three concepts: 1) use of quadrant sequences instead of individual quadrant points, 2) use of pressure quadrant method as a more reliable approach for identifying threshold conditions, and 3) coupling of velocity and pressure quadrant sequences to better resolve prevailing flow characteristics and associated coherent structures responsible for particle movement. This sequence-based approach highlights the importance of turbulence event duration and the dynamic process of particle dislodgement.

Statistical analysis of 105 dislodgement events, which are determined analytically, indicates the velocity quadrant sequences of $\left\{Q_{4 V}, Q_{1 V}, Q_{4 V}\right\}$ and $\left\{Q_{1 V}, Q_{4 V}\right\}$ as the most frequent flow processes present during the periods of dislodgement. The causative impact upon particle dislodgement is attributed to consecutive $\left\{Q_{1 P}, Q_{4 P}, Q_{1 P}\right\}$ or consistent $\left\{Q_{1 P}\right\}$ sequences. As for the relevant coherent structures, ensemble-average analysis identifies large-scale flow patterns characterized by sustained $Q_{4 V}$ events. The associated hydrodynamic effects are reflected by pertinent $Q_{1 P}$ over time. This result suggests that large-scale sweeps can generate excessive and persistent force fluctuations, which, in turn, promote conditions favorable for particle dislodgement. 
Overall, the turbulence sequence methods presented here can resolve dynamic processes of particle dislodgement more appropriately than the typical considerations of single instantaneous parameters. Furthermore, they support the impulse criterion as the proper way of accounting for the cumulative effect of coherent structures on the mobility of a particle.

\section{Appendix A: Perfect-rolling simulation}

The schematic of the forces applied on the test particle is shown in Fig. 1b. The equations of motion used to simulate the trajectory of a perfect-rolling spherical particle are based on Schmeeckle and Nelson [38]:

$r\left(\frac{7}{5} \rho_{s}+C_{m} \rho\right) \Gamma \frac{d^{2} \theta}{d t^{2}}=F_{D} \sin (\theta-\alpha)+F_{L} \cos (\theta-\alpha)-F_{G} \cos (\theta)$,

$r\left(\rho_{s}+C_{m} \rho\right) \Gamma\left(\frac{d \theta}{d t}\right)^{2}+F_{N}=F_{L} \sin (\theta-\alpha)-F_{D} \cos (\theta-\alpha)-F_{G} \sin (\theta)$,

where $\rho_{s}$ is the density of sphere, $C_{m}$ is the added mass coefficient, and $\Gamma$ is the sphere volume.

During the rolling process the applied forces are expected to change due to the varying relative speed between the flow and moving particle. The modulated drag force $F_{D m}$ can be determined as follows:

$F_{D m}=F_{D}\left(1-u_{P} / u\right)^{2}$,

where $u_{P}$ is the streamwise component of particle speed. The mean particle speed $(1.84 \mathrm{~cm} / \mathrm{s})$ reported here is $5.1 \%$ of the mobilizing flow velocity, causing $10 \%$ reduction in the drag force. However, the increasing degree of particle exposure $\left(A_{D}\right)$, from $87 \%$ to $98 \%$, compensates for the above force reduction. The consequent force variation is negligible $(\sim 2 \%)$. Also ignored are the modulations of the lift force and ambient flow velocity for the reason of simplicity.

During the 15-min pressure record, permanent particle displacement beyond the pivot point occurs intermittently at a mean frequency of 7.0 dislodgements $/ \mathrm{min}$. This result exhibits an error $<1 \%$ compared to the mobile-particle experiments performed by Celik et al. [16] under practically identical hydrodynamic conditions. The periods of these particle dislodgements range 
631 from 0.128 to $0.480 \mathrm{~s}$ with a mean value of $\sim 0.2 \mathrm{~s}$. Reasonable agreement is found in comparisons with other experimental results using particles of similar size $[14,15]$.

\section{Appendix B: Convergence tests}

634

635

636

637

638

639

640

641

642

643 644

645

646

647

648

649

650

651

652

Convergence tests are performed in advance to determine an appropriate temporal-resolution for the ensemble-average analysis with limited number of dislodgement replicates. The target variables are ensemble-averaged with $N$ replicates over a time interval $\Delta t$, which is assigned values ranging from 0.004 to $1.0 \mathrm{~s}$. Based on the variable convergence, the temporal-resolution of $\Delta t=0.04 \mathrm{~s}$ can maximize the turbulence-resolving capacity while maintaining stationary statistics with $N<100$ replicates. Fig. B.1 shows the convergence of $u$-velocity and quadrant types $Q_{4 V}$ and $Q_{1 V}$ using the above resolution. The degree of $u$ variation is progressively reduced and the standard deviation is $<2 \%$ of the mean velocity at $N=90$ (Fig. B.1a). The velocity quadrants can converge even faster than $u$-velocity (Fig. B.1b). With the same time-resolution, the pressure data yield very similar results in the convergence tests (not presented).
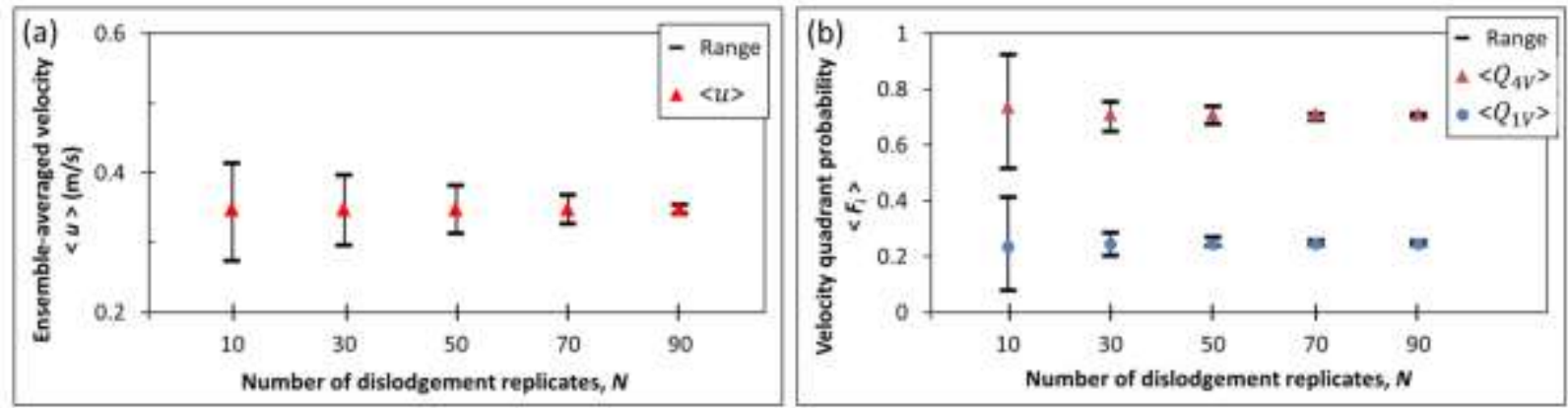

Fig. B.1 Convergence test for the ensemble-average analysis: (a) $u$-velocity and (b) dominant velocity quadrants $Q_{4 V}$ and $Q_{1 V}$.

Acknowledgement The support of the National Science Foundation (grant CBET-1033196) for this study is gratefully acknowledged. We also appreciate the comments and suggestions provided by four reviewers and the associate editor in improving the overall presentation of this work and for sharpening a number of points raised here.

\section{References}


[1] Best J. On the entrainment of sediment and initiation of bed defects: insights from recent developments within turbulent boundary layer research. Sedimentology 1992; 39(5): 797-811.

[2] Kaftori D, Hetsroni G, Banerjee S. Particle behavior in the turbulent boundary layer. I. Motion, deposition, and entrainment. Physics of Fluids 1995; 7(5): 1095-106.

[3] Smart G, Habersack H. Pressure fluctuations and gravel entrainment in rivers. Journal of Hydraulic Research 2007; 45(5): 661-73.

[4] Vowinckel B, Kempe T, Fröhlich J. Fluid-particle interaction in turbulent open channel flow with fullyresolved mobile beds. Advances in Water Resources 2014; 72: 32-44.

[5] Wallace JM. Quadrant Analysis in Turbulence Research: History and Evolution. Annual Review of Fluid Mechanics 2016; 48: 131-58.

[6] Bennett SJ, Bridge JS, Best JL. Fluid and sediment dynamics of upper stage plane beds. Journal of Geophysical Research: Oceans 1998; 103(C1): 1239-74.

[7] Lelouvetel J, Bigillon F, Doppler D, Vinkovic I, Champagne JY. Experimental investigation of ejections and sweeps involved in particle suspension. Water resources research 2009; 45(2).

[8] Schmeeckle MW. Numerical simulation of turbulence and sediment transport of medium sand. Journal of Geophysical Research: Earth Surface 2014; 119(6): 1240-62.

[9] Celik AO, Diplas P, Dancey CL. Instantaneous turbulent forces and impulse on a rough bed: Implications for initiation of bed material movement. Water Resources Research 2013; 49(4): 2213-27.

[10] Heathershaw A, Thorne P. Sea-bed noises reveal role of turbulent bursting phenomenon in sediment transport by tidal currents. Nature 1985; 316: 339-42.

[11] Nelson JM, Shreve RL, McLean SR, Drake TG. Role of near-bed turbulence structure in bed load transport and bed form mechanics. Water Resources Research 1995; 31(8): 2071-86.

[12] Schmeeckle M. The role of velocity, pressure, and bed stress fluctuations in bed load transport over bed forms: numerical simulation downstream of a backward-facing step. Earth Surface Dynamics 2015; 3(1): 105.

[13] Paintal A. A stochastic model of bed load transport. Journal of Hydraulic Research 1971; 9(4): $527-$ 54.

[14] Valyrakis M, Diplas P, Dancey CL. Entrainment of coarse particles in turbulent flows: An energy approach. Journal of Geophysical Research: Earth Surface 2013; 118(1): 42-53.

[15] Wu FC, Shih WR. Entrainment of sediment particles by retrograde vortices: Test of hypothesis using near-particle observations. Journal of Geophysical Research: Earth Surface 2012; 117(F3).

[16] Celik AO, Diplas P, Dancey CL, Valyrakis M. Impulse and particle dislodgement under turbulent flow conditions. Physics of Fluids 2010; 22(4): 046601.

[17] Diplas P, Dancey CL, Celik AO, Valyrakis M, Greer K, Akar T. The role of impulse on the initiation of particle movement under turbulent flow conditions. Science 2008; 322(5902): 717-20.

[18] Nelson JM, Schmeeckle MW. Entrainment revisited: high-frequency measurements of forces on sediment particles in turbulent flow. Proceedings of the 7th Federal Interagency Sedimentation Conference 2001; XI: 110-7.

[19] Valyrakis M, Diplas P, Dancey CL, Greer K, Celik AO. Role of instantaneous force magnitude and duration on particle entrainment. Journal of Geophysical Research: Earth Surface 2010; 115(F2).

[20] Hofland B, Booij R. Measuring the flow structures that initiate stone movement. River flow2004. pp. 821-30.

[21] Dwivedi A, Melville B, Raudkivi AJ, Shamseldin AY, Chiew Y-M. Role of turbulence and particle exposure on entrainment of large spherical particles in flows with low relative submergence. Journal of Hydraulic Engineering 2012; 138(12): 1022-30.

[22] Dwivedi A, Melville BW, Shamseldin AY, Guha TK. Flow structures and hydrodynamic force during sediment entrainment. Water Resources Research 2011; 47(1). 
[23] Keylock CJ, Lane SN, Richards KS. Quadrant/octant sequencing and the role of coherent structures in bed load sediment entrainment. Journal of Geophysical Research: Earth Surface 2014; 119(2): 264-86. [24] Einstein HA, El-Samni E-SA. Hydrodynamic forces on a rough wall. Reviews of modern physics 1949; 21(3): 520.

[25] Celik AO, Diplas P, Dancey CL. Instantaneous pressure measurements on a spherical grain under threshold flow conditions. Journal of Fluid Mechanics 2014; 741: 60-97.

[26] Schmeeckle MW, Nelson JM, Shreve RL. Forces on stationary particles in near-bed turbulent flows. Journal of Geophysical Research: Earth Surface 2007; 112(F2).

[27] Hofland B, Battjes JA, Booij R. Measurement of fluctuating pressures on coarse bed material. Journal of Hydraulic Engineering 2005; 131(9): 770-81.

[28] Kalinske A. Movement of sediment as bed load in rivers. Eos, Transactions American Geophysical Union 1947; 28(4): 615-20.

[29] Hardy RJ, Best JL, Lane SN, Carbonneau PE. Coherent flow structures in a depth-limited flow over a gravel surface: The role of near-bed turbulence and influence of Reynolds number. Journal of geophysical research: earth surface 2009; 114(F1).

[30] Robert A, Roy A, De Serres B. Turbulence at a roughness transition in a depth limited flow over a gravel bed. Geomorphology 1996; 16(2): 175-87.

[31] Shields A. Application of similarity principles and turbulence research to bed-load movement. Soil Conservation Service1936.

[32] Fenton J, Abbott J. Initial movement of grains on a stream bed: The effect of relative protrusion. Proceedings of the Royal Society of London A: Mathematical, Physical and Engineering Sciences. The Royal Society1977. pp. 523-37.

[33] Pope SB. Turbulent Flows. Cambridge: Cambridge Univ Press 2001.

[34] Vassilicos JC. Dissipation in turbulent flows. Annual Review of Fluid Mechanics 2015; 47: 95-114.

[35] Amir M, Nikora VI, Stewart MT. Pressure forces on sediment particles in turbulent open-channel flow: a laboratory study. Journal of Fluid Mechanics 2014; 757: 458.

[36] Kassem H, Thompson CE, Amos CL, Townend IH. Wave-induced coherent turbulence structures and sediment resuspension in the nearshore of a prototype-scale sandy barrier beach. Continental Shelf Research 2015; 109: 78-94.

[37] Mignot E, Hurther D, Barthelemy E. On the structure of shear stress and turbulent kinetic energy flux across the roughness layer of a gravel-bed channel flow. Journal of Fluid Mechanics 2009; 638: 42352.

[38] Schmeeckle MW, Nelson JM. Direct numerical simulation of bedload transport using a local, dynamic boundary condition. Sedimentology 2003; 50(2): 279-301.

[39] McEwan I, Heald J. Discrete particle modeling of entrainment from flat uniformly sized sediment beds. Journal of Hydraulic Engineering 2001; 127(7): 588-97.

[40] Wu FC, Chou Y-J. Rolling and lifting probabilities for sediment entrainment. Journal of Hydraulic Engineering 2003; 129(2): 110-9.

[41] Nezu I, Nakagawa H. Turbulence in Open-Channel Flows. A A Balkema, Rotterdam, Netherlands 1994.

[42] Wu Y, Christensen KT. Population trends of spanwise vortices in wall turbulence. Journal of Fluid Mechanics 2006; 568: 55-76.

[43] Chien N, Wan Z. Mechanics of Sediment Transport. ASCE, Reston, Virginia, USA, 1999.

[44] Diplas P, Dancey CL. Coherent flow structures, initiation of motion, sediment transport and morphological feedbacks in rivers. Coherent Flow Structures at Earth's Surface 2013: 289-307. 
746 [45] Wiberg PL, Smith JD. Calculations of the critical shear stress for motion of uniform and 747 heterogeneous sediments. Water resources research 1987; 23(8): 1471-80. 